\title{
Limb Salvage Surgery in Fungating Giant Cell Tumors: A Report of Three Cases
}

\author{
Namith Rangaswamy ${ }^{1}$, Venkatesan Sampath Kumar ${ }^{1}$, Roshan Banjara ${ }^{1}$, Abdul Majeed ${ }^{1}$, Devansh \\ Goyal $^{1}$, Shah Alam Khan ${ }^{1}$
}

\section{Learning Point of the Article:}

Endoprosthetic reconstruction and limb salvage surgeries are feasible in fungating giant cell tumors of extremities provided oncological principles are adhered to.

\section{Abstract}

Introduction: The WHO defines giant cell tumor as a benign locally aggressive neoplasm with metastasizing capacity and aggressive behavior. Very rarely, these tumors are seen fungating, mostly when neglected. But when they do, the treatment option commonly conferred is amputation of the limb which is disabling and traumatizing.

Case Report: We report three cases of fungating limb masses (proximal tibia, distal fibula, and distal radius) diagnosed with giant cell tumor histologically, undergoing limb saving surgeries with various reconstruction techniques to endorse a good quality of life and functioning limb.

Conclusion: Our study is one of the earliest to report medium-term follow-up after such limb salvage procedure. We recommend that salvage procedures should be considered in giant cell tumors even in the presence of fungation if there is no neurovascular encasement.

Keywords: Giant cell tumor, fungation, limb salvage surgery, endoprosthesis, mesh reconstruction.

\section{Introduction}

Giant cell tumors were described as early as in 1818 by Cooper and Travers [1]. GCT constitutes about $5 \%$ of all bone tumors and $20 \%$ of benign bone tumors. Eighty percent of GCTs are found to occur between 20 and 50 years of age, having peak incidence at the 3 rd decade and they uncommonly present with fungation. Microscopically, giant cell tumors are a blend of spindle-shaped stromal cells, multinucleated giant cells resembling osteoclasts, and mononuclear cells which are positive for CD-68 [2]. GCT has a well-defined non-sclerotic margin located eccentrically in long bone extending close to the articular surface in patients with closed epiphysis seldom crossing the joint [1]. Conventionally treatment of GCT included surgical curettage and PMMA (polymethylmethacrylate) cement application with or without adjuvant chemical ablation. Recent advances have shown that chemotherapeutic drugs such as denosumab have shown dramatic response with tumor necrosis in cases of early GCT [1]. However, wide excision of the tumor is recommended in large or recurrent tumors. Radical amputation procedures are still a widely practiced treatment for massive tumors ulcerating the skin, leading to high morbidity and severe disability. Little or no literature of experience is available currently on limb salvage surgeries in such cases of fungating tumors. We report a series of three cases with fungating limb masses with histopathological diagnosis of giant cell tumor undergoing limb saving surgical procedures. Informed written consent was obtained from all the patients explaining the possibility of publication.

\section{Case Report}

\section{Case 1}

A 21-year-old female who presented to our outpatient clinic with complaints of pain and swelling in her left knee for 8

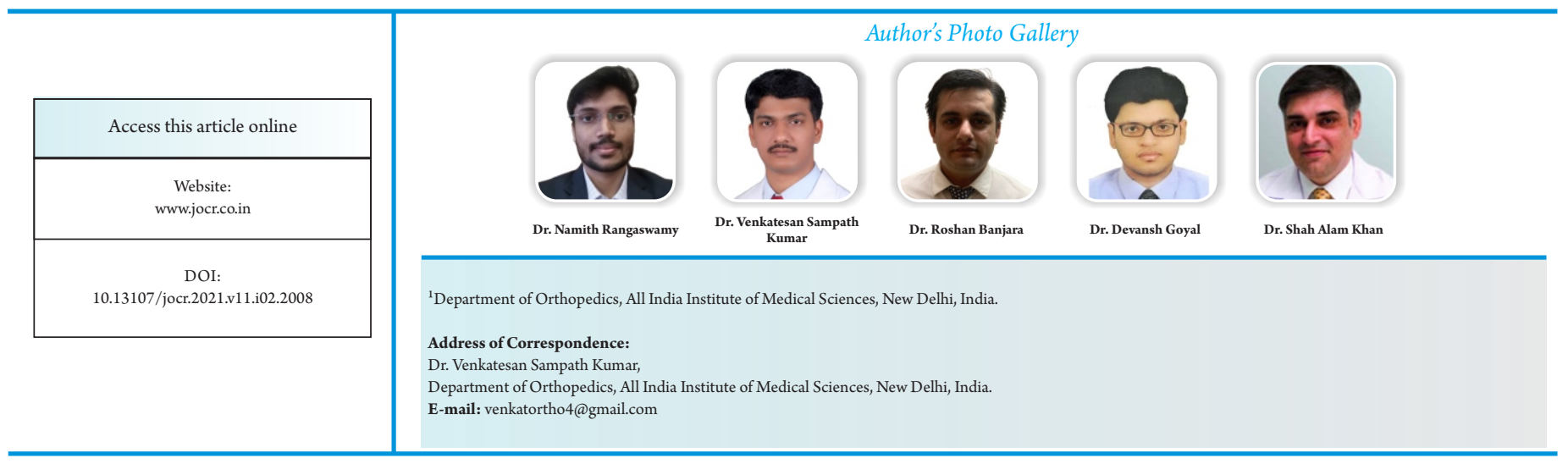

Journal of Orthopaedic Case Reports | pISSN 2250-0685 | eISSN 2321-3817 | Available on www.jocr.co.in | doi:10.13107/jocr.2021.v11.i02.2008 This is an Open Access article distributed under the terms of the Creative Commons Attribution Non-Commercial License (http://creativecommons.org/licenses/by-nc/3.0) which permits unrestricted non-commercial use, distribution, and reproduction in any medium, provided the original work is properly cited. 


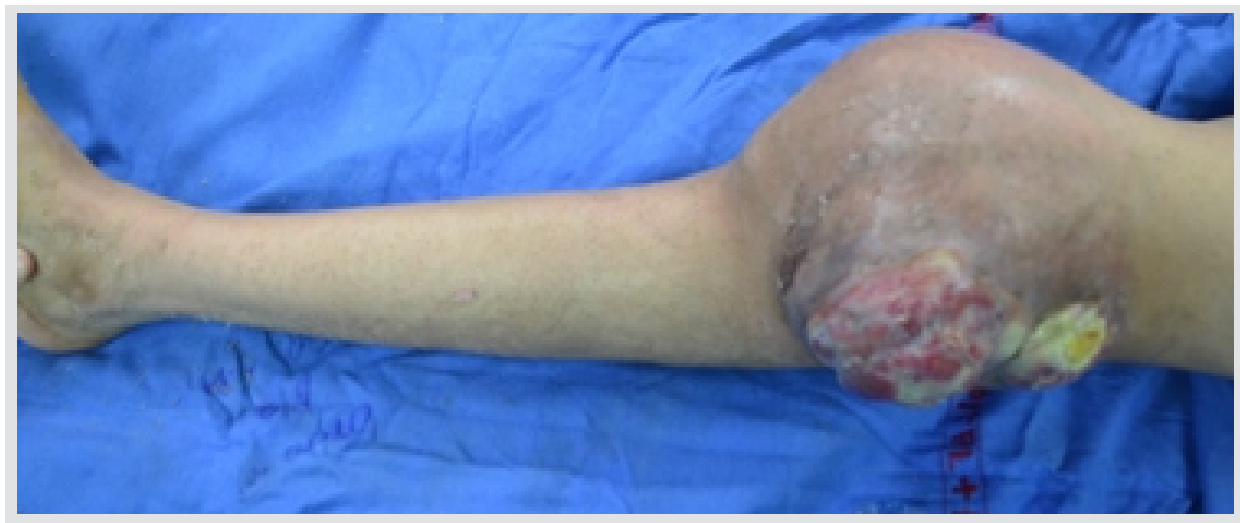

Figure 1: Pre-operative clinical photograph showing a fungating mass of the left knee.

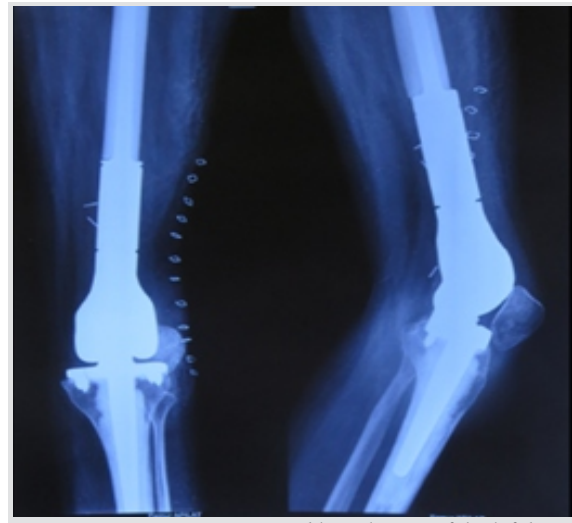

Figure 2: Post-operative AP and lateral X-ray of the left kne with distal femoral endoprosthesis. months before her first visit, with a sudden increase in size for later 2 months. On examination of her limb, there was gross wasting of quadriceps muscle with diffuse swelling over the lateral aspect of the distal thigh measuring approximately $12 \mathrm{~cm}$ long and $5 \mathrm{~cm}$ across. The local temperature was raised with tenderness on deep palpation restricting terminal knee flexion. On anteroposterior and lateral Roentgenogram, there was an eccentric lytic and sclerotic lesion in the left distal end femur with a narrow zone of transition without evidence of joint involvement, with some areas of soft-tissue calcification and minimal periosteal reaction. Core biopsy done reported to have cellular tissues with multinucleated osteoclast-like giant cells admixed with mononuclear cells, round to oval to spindleshaped without significant cellular atypia and histological diagnosis of GCT was made.

The patient was lost to follow up, presented 3 months later with an enlarged mass measuring $15 \mathrm{~cm} \times 12 \mathrm{~cm}$ with ulceration over the lateral aspect of the knee (Fig. 1). New AP and the lateral radiograph showed a large destructive lesion in the distal femur with chondroid matrix, broad zone of transition with break-in cortex, and destruction of joint margin, fresh magnetic resonance images showed a heterogeneous mass of the left distal femur with the erosion of joint margin extending about $15 \mathrm{~cm}$ from the joint line without involvement of femoral or popliteal vessels and nerves. CT scan of the chest was found to be normal. A final diagnosis of fungating non-metastatic giant cell tumor of the left distal femur, Campanacci Grade III with the left knee joint involvement was made.

Management options were explored, preferably to excise the tumor and reconstruct the limb to provide the functional ability, and the decision of wide excision with endoprosthesis application was made. Intraoperatively, careful dissection was done protecting the neurovascular bundle and the tumor was removed preventing any soft-tissue spillage. After confirmation of the safe margin through the frozen section examination, distal femoral endoprosthesis was cemented (Fig. 2). The postoperative specimen had features of GCT without atypia with safe bony and soft-tissue margins. The patient was put on protected weight-bearing initially, slowly progressing to full weight-bearing along with knee range of movement and quadriceps strengthening exercises. At follow-up of 2 years with extensive physical therapy, the patient has good functional results with flexion of $90^{\circ}$ (Fig. 3) and is walking without pain enabling her to carry on comfortably with routine activities. No evidence of infection or local recurrence was seen.

\section{Case 2}

A 28-year-old male patient presented with a bleeding mass from the left distal leg which developed following curettage elsewhere for a suspected giant cell tumor of the left distal fibula 1 year earlier. On examination, we found a mass of $8 \times 8 \mathrm{~cm}$ with

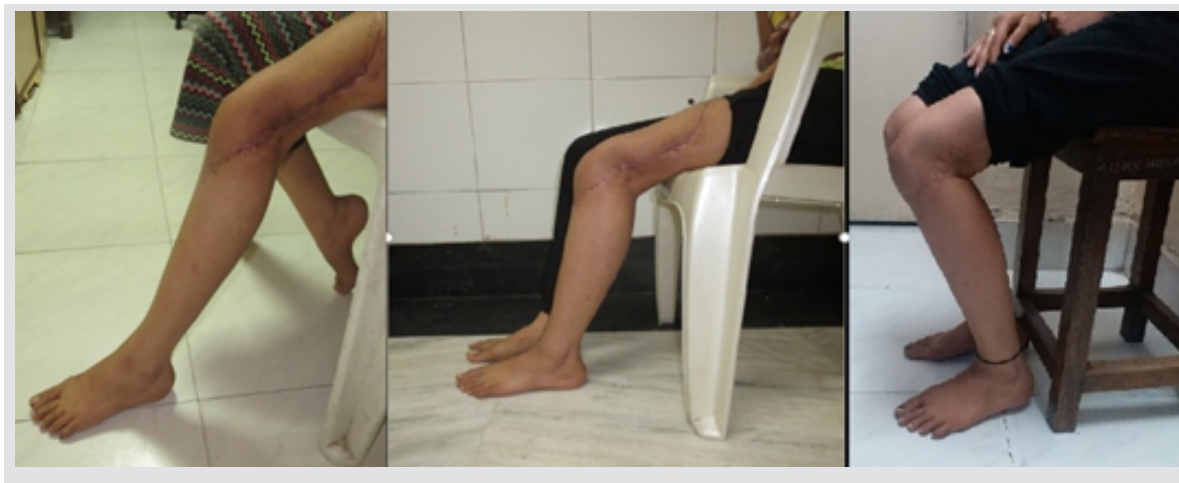

Figure 3: Serial post-operative photographs of a patient with gain in knee range of movement and a functional limb.

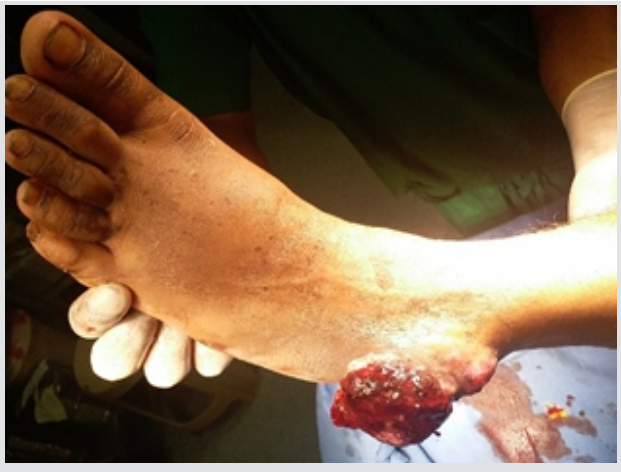

Figure 4: Pre-operative clinical photograph of fungating mass over the left ankle arising from distal fibula. 


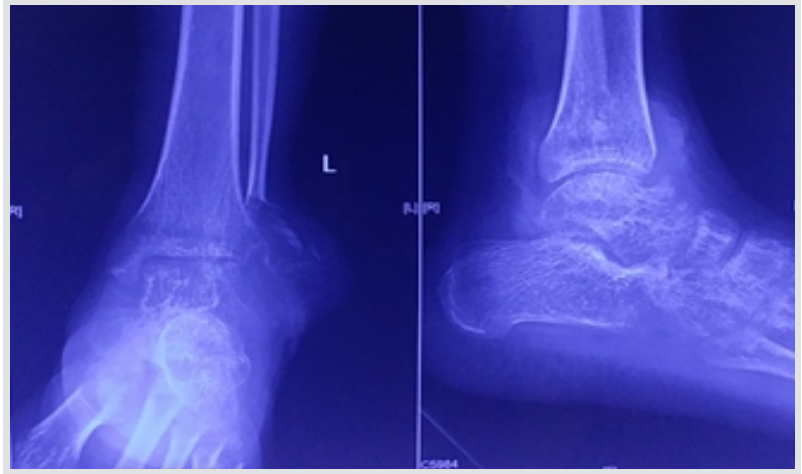

Figure 5: Antero-posterior and lateral X-ray of the left ankle showing lytic destructive lesion of the left distal fibula.

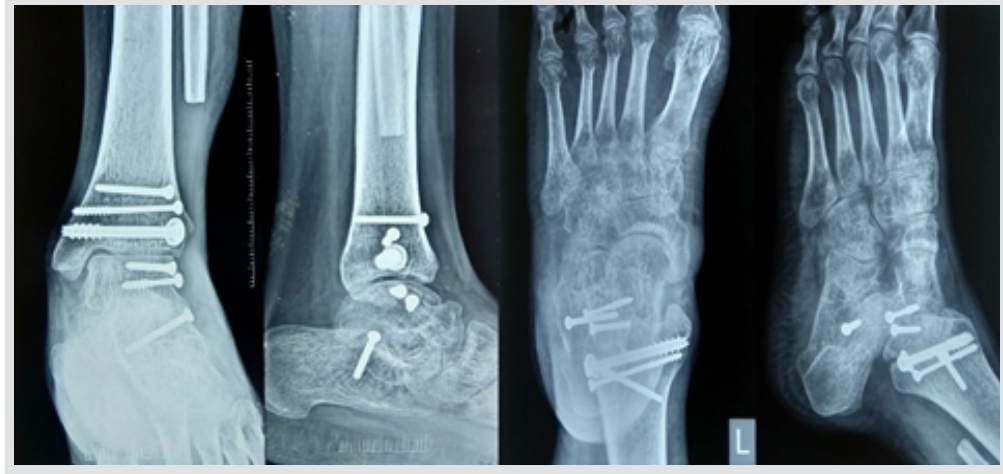

Figure 6: Post-operative antero-posterior (AP) and lateral view radiograph of the left ankle and AP and oblique view of a left foot showing resected distal fibula with reconstruction using mesh and screws, fixed into the distal tibia, talus, and calcaneum. a large bleeding raw area over the lateral malleolus (Fig. 4). Imaging showed a lytic destructive lesion of distal fibula extending into the soft tissue on X-ray radiography (Fig. 5) with a substantiating picture on MRI. The diagnosis was confirmed by histopathological examination of biopsy specimen as GCT. The limb salvage procedure was done by wide resection of the tumor. Mesh repair was done to stabilize the ankle with screws placed in the distal tibia, talus, and calcaneum (Fig. 6). Belowknee slab was provided and gradual mobilization protocol was followed to get a successful mobile limb. No post-operative complication was encountered. At lyear follow-up, the patient can walk full weight-bearing unassisted with satisfactory ankle ROM (Fig. 7).

\section{Case 3}

A 38-year-old male having a swelling in his left wrist for 3 years for which he was advised amputation below the level of his elbow elsewhere and that drew him to our institution. On presentation, physical examination revealed a dumbbell-shaped mass measuring $12 \times 10 \mathrm{~cm}$ over his left wrist with extensively stretched skin and fungation over the dorsal surface (Fig. 8). Roentgenogram showed an expansile lytic lesion with soft- tissue extension over the distal radius with typical soap bubble appearance (Fig. 9) in concordance with MR imaging of heterogeneous $\mathrm{T} 2 \mathrm{~W}$ hyperintense lesion with an air-fluid level. The biopsy confirmed the diagnosis of GCT. Surgical intervention was done with careful resection of the tumor with meticulous dissection to save multiple encased tendons along with the centralization of the ulna with wrist arthrodesis plate to fuse the wrist joint and immobilized with a splint (Fig. 10a). Due to uncontrolled Type 2 diabetes mellitus, the patient developed deep surgical site infections in the early postoperative period. Plate removal with thorough surgical debridement was done and stabilized with K-wire (Fig. 10b) along with splinting. Adequate glycemic control with culture appropriate antibiotic was administered and healthy wound healing with successful arthrodesis was achieved to obtain a good functioning limb at the final follow-up of 1 year.

\section{Discussion}

Giant cell tumor is a locally aggressive benign tumor [3] commonly involving the end of a long bone in young adults between 20 and 40 years of age with female predominance [4]. Being a benign tumor, GCT can rarely be lethal, but is usually

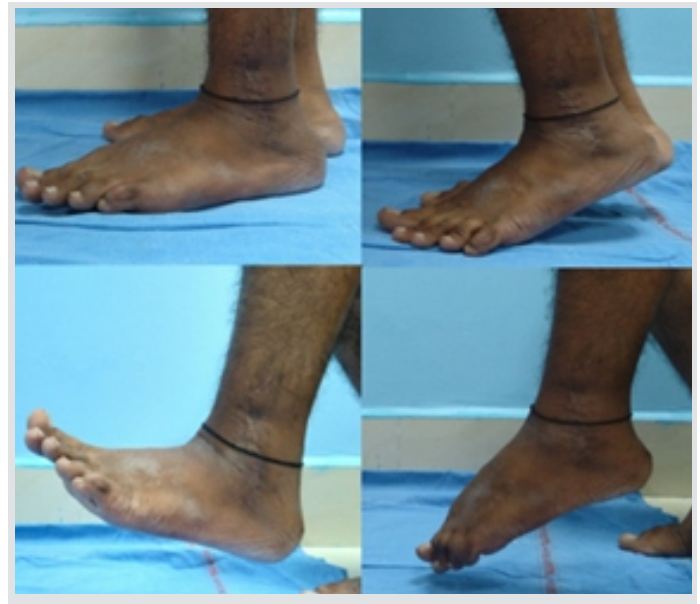

Figure 7: Post-operative photographs of the left ankle showing healthy surgical scar with complete recovery of range of movement and functional ability.

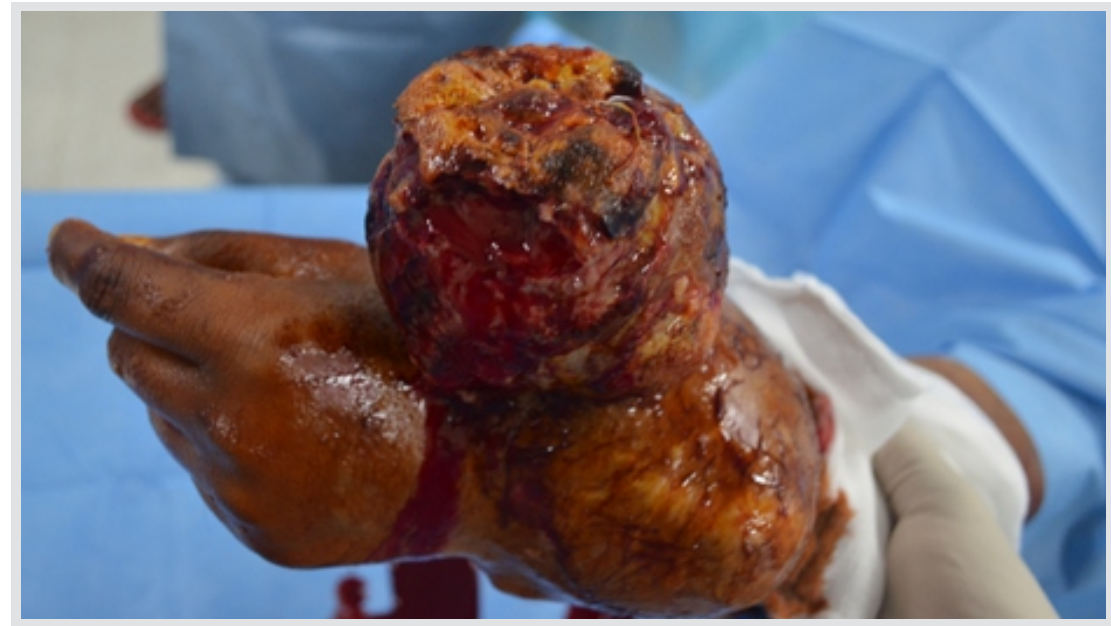

Figure 8: Clinical radiograph of huge dumbbell-shaped mass from the left wrist with a fungating and bleeding surface with areas of necrosis. 


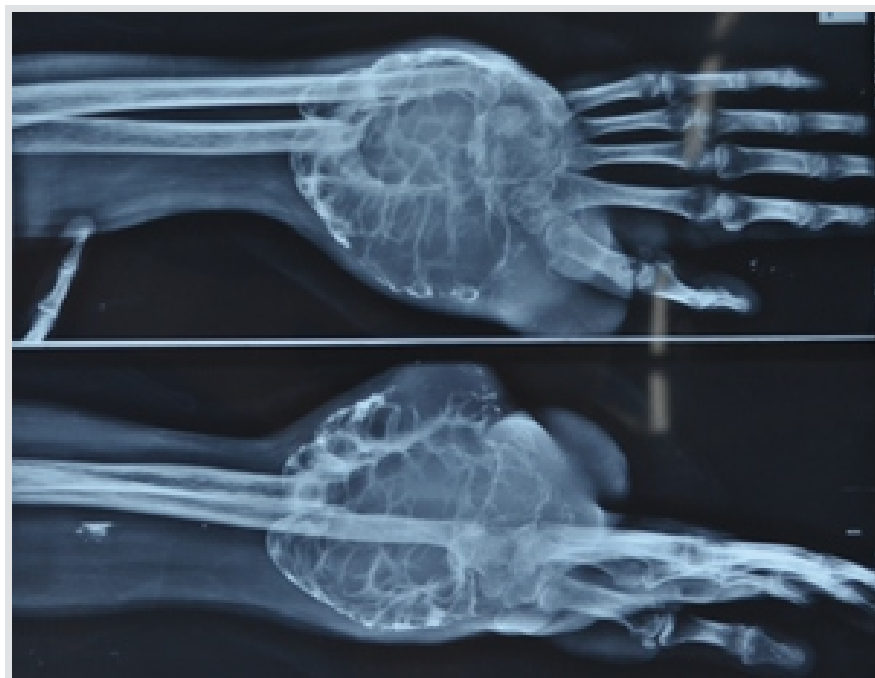

Figure 9: Pre-operative radiograph of the left wrist showing expansile lytic lesion arising from th distal radius with typical soap bubble appearance.

associated with the destruction of local bony architecture and can prove distressing due to periarticular locations. The ideal treatment solution is still debatable with a huge spectrum of surgical options available, the principle of treatment is to eradicate the tumor and preserve the function of a limb [5]. However, even in current practice, radical procedures leading to the removal of limbs have been the main consensus of management in fungating, neglected, and large tumors. Neglected orthopedic malignancies have been a challenge to confront for surgeons worldwide. Some of the main reasons for patients to present in advanced stages are neglect by the patient and family, low socioeconomic status, unawareness, lack of access to medical facilities, and even misdiagnosis sometimes, leading to an enormous rise in morbidity and mortality [6]. The experience of amputation can be traumatizing [7]. Although there is a paucity of studies on quality of life following major limb amputation, Blåder et al. reported on 35 patients with malignant upper limb tumors who underwent amputation, half had to loose occupation affecting social life [8].

The literature of experience on limb salvage procedures in fungating tumors of bone are very few and limited to case series, and none involve implantation of the endoprosthesis to provide a completely functional limb. Kamal et al. in his case report presented a case of 4 years lost to follow-up patient with a malignant giant cell tumor of the patella, presenting with an ulcerating lump over the left knee without the involvement of joint, for which complete excision of the tumor and reconstruction of extensor mechanism were done to obtain a mobile limb [9]. Shrestha et al. presented a case of fungating giant cell tumor with the complete destruction of lower end ulna with the involvement of the wrist joint where the limb was saved by wide excision of the tumor with arthrodesis of the wrist joint [10]. Chinder et al. recently published a case report on Ewing's sarcoma of scapula presenting with fungation where

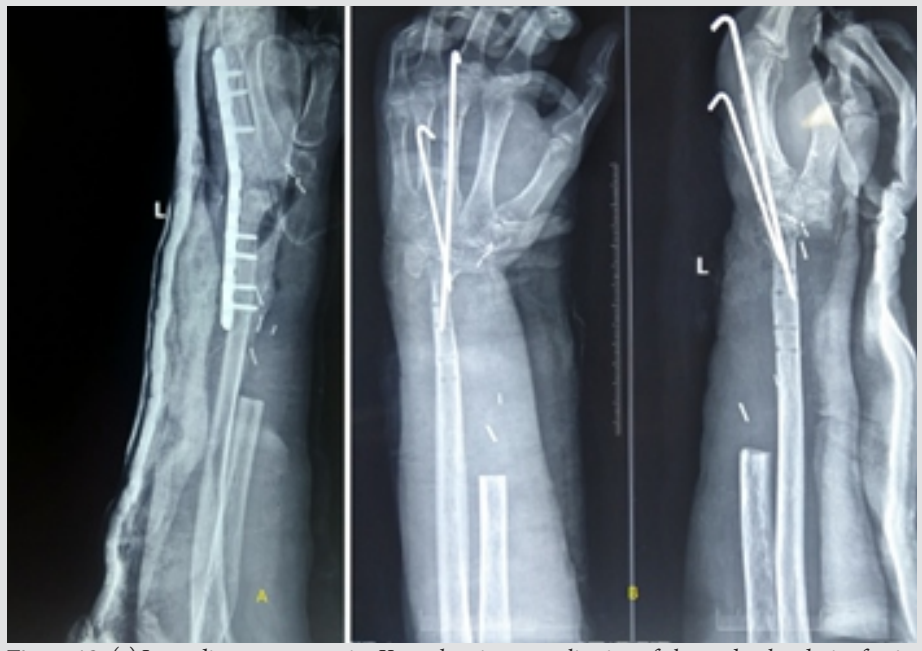

Figure 10: (a) Immediate post-operative $\mathrm{X}$-ray showing centralization of ulna and arthrodesis of wris joint with arthrodesis plate, (b) radiograph following debridement and plate removal with K-wire stabilization for deep surgical site infection.

they managed to salvage the limb [11].

Fungating tumors make for $35 \%$ of cases undergoing amputation among orthopedic tumors [12]. The decision to perform limb salvage surgery was taken in all the three patients in our report because the predominant neurovascular bundle was uninvolved by the tumor and the patients were unwilling for amputation. Since GCT is a benign tumor, even if the tumor has to recur locally in the soft tissues, it can be safely managed with wide excision without any increased risk of mortality. Amputation should be reserved and considered only when necessary to achieve palliation of debilitating symptoms as it results in severe disability of function, hugely affecting the livelihood and quality of life.

\section{Conclusion}

The primary concern in the management of fungating tumors is the fear of infection. Our patients managed with endoprosthetic reconstruction and synthetic mesh reconstruction had no signs of post-operative infection. However, a diabetic patient who underwent arthrodesis developed surgical site infection requiring debridement and resurgery. Hence, we believe systemic factors together with the local nature of the disease contribute to the successful outcome of the management. Therefore, fungation should not be the sole deciding element for amputation.

\section{Clinical Message}

Limb salvage surgery should be considered in fungating tumors as long as oncological margins are respected. 


\section{References}

1. Chakarun CJ, Forrester DM, Gottsegen CJ, Patel DB, White EA, Matcuk GR. Giant cell tumor of bone: Review, mimics, and new developments in treatment. Radiographics 2013;33:197-211.

2. Beebe-Dimmer JL, Cetin K, Fryzek JP, Schuetze SM, Schwartz K. The epidemiology of malignant giant cell tumors of bone: An analysis of data from the surveillance, epidemiology and end results program. Rare Tumors 2009; 1:e52.

3. Fletcher CD, Unni KK, Mertens F, World Health Organization, International Agency for Research on Cancer, editors. Pathology and Genetics of Tumours of Soft Tissue and Bone. Lyon: IARC Press, World Health Organization; 2002.

4. Gupta R, Viswanathan S, Jambhekar NA, Prabhudesai S, Merchant N, Puri A, et al. Clinicopathologic profile of 470 giant cell tumors of bone from a cancer hospital in Western India. Ann Diagn Pathol 2008; 12:239-48.

5. Sobti A, Agarwal P, Agarwala S, Agarwal M. Giant cell tumor of bone-an overview. Arch Bone Jt Surg 2016;4:2-9.

6. Siddiqui Y, Sherwani M, Khan A, Zahid M, Abbas M, Asif N. Neglected orthopedic oncology-causes, epidemiology and challenges for management in developing countries. Indian J Cancer 2015;52:325-9.

7. Sahu A, Sagar R, Sarkar S, Sagar S. Psychological effects of amputation: A review of studies from India. Ind Psychiatry J2016;25:4-10.

8. Blåder S, Gunterberg B, Markhede G. Amputation for tumor of the upper arm. Acta Orthop Scand 1983;54:226-9.

9. Kamal AF, Hutagalung EU, Gumay S, Prabowo Y, Yanuarso Y. Primary malignant giant cell tumor of the patella: Report of a rare case. Med J Indones 2013;22:238-42.

10. Shrestha B, Kumar P, Singh GJ, Singh MP. Fungating primary locally malignant giant cell tumor of ulna-a case report.JNMAJ Nepal Med Assoc 2008;47:41-3.

11. Chinder P, Hindiskere S, Doddarangappa S, Pal U, Daggupati KT. Massive fungating Ewing's sarcoma of scapula treated by limb salvage surgery: A case report. J Orthop Case Rep 2019;9:58-61.

12. Parsons CM, Pimiento JM, Cheong D, Marzban SS, Gonzalez RJ, Johnson D, et al. The role of radical amputations for extremity tumors: A single institution experience and review of the literature. J Surg Oncol 2012;105:149-55.

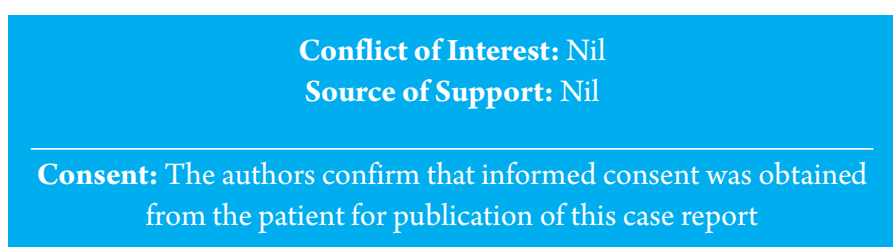

How to Cite this Article

Rangaswamy N, Kumar VS, Banjara R, Majeed A, Goyal D, Khan SA. Limb salvage surgery in fungating giant cell tumors: A report of three cases. Journal of Orthopaedic Case Reports 2021 February;11(2): 19-23. 